\title{
Inversion Formula for the Discrete Radon Transform
}

\author{
Takeshi KAWAZOE
}

Keio University

\begin{abstract}
We shall give a characterization of the discrete Radon transform of functions in the Schwartz space on $\mathbf{Z}^{n}$ and obtain various inversion formulas for the discrete Radon transform on $\mathbf{Z}^{2}$.
\end{abstract}

\section{Introduction}

The classical Radon transform was firstly defined on $\mathbf{R}^{2}$ by J. Radon [5] as the integral over a line $L$ in $\mathbf{R}^{2}$ :

$$
R f(L)=\int_{L} f(x) d \mu(x)
$$

where $d \mu(x)$ is the Euclidean measure on $L$. Each line $L$ with the direction vector $\omega \in S^{1}$ is given by $L(\omega, t)=\left\{x \in \mathbf{R}^{2} \mid x \cdot \omega=t\right\}$ where $t \in \mathbf{R}$ and $x \cdot \omega$ is the inner product of $x$ and $\omega$. Hence the set of all lines in $\mathbf{R}^{2}$ is parameterized as $S^{1} \times \mathbf{R} /\{ \pm 1\}$. The Radon transform $R$ is related to the Fourier transform as the slice formula:

$$
\widetilde{R f}(L(\omega, \cdot))(\lambda)=\widetilde{f}(\lambda \omega),
$$

where the left hand side is the one-dimensional Fourier transform and the right hand side is the two-dimensional one. Hence we can recover $f$ from $R f$ by using this relation. However, this inversion formula has a difficulty of convergence of inversion Fourier transforms. Another method to invert the Radon transform involves the dual Radon transform. We integrate $R f(L(\omega, t))$ over $S^{1}$ and apply a fractional differential operator on $\mathbf{R}$ such as $\sqrt{-\Delta}$. The idea to recover a function on $\mathbf{R}^{2}$ from its integrals over all lines is generalized in different settings by various people. For an extensive survey we refer to Helgason's book [4].

In this paper, as analogue of the classical Radon transform on $\mathbf{R}^{2}$, we shall consider the discrete Radon transform on $\mathbf{Z}^{n}$, which was originally proposed by Strichartz [6] and was

Received December 9, 2013; revised May 3, 2014 2000 Mathematics Subject Classification: 44A12

Key words and phrases: discrete Radon transform, Schwartz space, inversion formula

The author is partly supported by Grant-in-Aid for Scientific Research (C), No. 24540191, Japan Society for the Promotion of Science. 
introduced by Abouelaz and Ihsane [1]. For a function $f$ on $\mathbf{Z}^{n}$ the discrete Radon transform $R f$ is defined by the summation of $f(m)$ over $m$ in a discrete hyperplane $H$ in $\mathbf{Z}^{n}$ :

$$
R f(H)=\sum_{m \in H} f(m) .
$$

Let $\mathbf{G}$ be the set of all discrete hyperplanes $H$ in $\mathbf{Z}^{n}$ (see §2). Then $R$ transfers functions on $\mathbf{Z}^{n}$ to ones on $\mathbf{G}$. Some basic properties of the discrete Radon transform $R$ were obtained in [1] and [2]. Especially, similarly as in the classical case, the slice formula and the inversion formula for $R$ were established. Roughly speaking, the one-dimensional Fourier series of $R f$ is related with the $n$-dimensional Fourier series of $f$ (see (4)). Since $\mathbf{G}$ is a discrete set, as the answer in [6], the inversion formula for $R$ has a quite simple form without a dual Radon transform and a fractional differential operator (see (5)). However, concerning the Schwartz theorem, we have only a partial result. In the classical case, the image of the Radon transform of Schwartz space $\mathcal{S}\left(\mathbf{R}^{2}\right)$ is characterized as functions $F$ in $\mathcal{S}\left(S^{1} \times \mathbf{R}\right)$ which have the property that for each $k=0,1,2, \ldots$,

$$
\int_{-\infty}^{\infty} F(\omega, t) t^{k} d t
$$

can be written as a homogeneous $k$ th degree polynomial of $\omega$ (see [3]). In our discrete case, if $f$ is a rapidly decreasing function on $\mathbf{Z}^{n}$, then $R f(H)$ is decreasing when $H$ goes away from the parallel hyperplane through the origin and the sum of $R f(H)$ over parallel hyperplanes satisfies the above property of a homogeneous polynomial. Hence $R$ maps injectively the Schwartz space $\mathcal{S}\left(\mathbf{Z}^{n}\right)$ into a kind of Schwartz classes on $\mathbf{G}$ satisfying these properties. But, this map is not surjective.

The aim of this paper is to give a more precise characterization of the image of the discrete Radon transform of $\mathcal{S}\left(\mathbf{Z}^{n}\right)$ and obtain several new inversion formulas for the discrete Radon transform on $\mathbf{Z}^{2}$.

\section{Notation}

We briefly state some basic properties on the discrete Radon transform $R$ on $\mathbf{Z}^{n}$. For more details we refer to [1] and [2].

Let $\mathcal{P}$ be the set of all $a=\left(a_{1}, a_{2}, \ldots, a_{n}\right) \in \mathbf{Z}^{n}$ such that the greatest common divisor $d\left(a_{1}, a_{2}, \ldots, a_{n}\right)$ equals 1 . For each $a \in \mathcal{P}$ and $k \in \mathbf{Z}$, the set $H(a, k)=\left\{x \in \mathbf{Z}^{n} \mid a x=k\right\}$ forms a discrete hyperplane in $\mathbf{Z}^{n}$, where $a x$ is the inner product of $a$ and $x$. Then the set $\mathbf{G}$ of discrete hyperplanes on $\mathbf{Z}^{n}$ is parameterized as $\mathcal{P} \times \mathbf{Z} /\{ \pm 1\}$ (see [1], §2). Hence $R$ in (1) transfers a function $f(m)$ on $\mathbf{Z}^{n}$ to $R f(H(a, k))$ on $\mathcal{P} \times \mathbf{Z} /\{ \pm 1\}$. Let $l^{p}\left(\mathbf{Z}^{n}\right), 1 \leq p<\infty$, denote the space of all functions $f$ on $\mathbf{Z}^{n}$ with finite $l^{p}$-norm

$$
\|f\|_{p}=\left(\sum_{m \in \mathbf{Z}^{n}}|f(m)|^{p}\right)^{1 / p}
$$


and $l^{\infty}\left(\mathbf{Z}^{n}\right)$ the one with finite $l^{\infty}$-norm. Since $\underset{k \in \mathbf{Z}}{\cup} H(a, k)=\mathbf{Z}^{n}$ for all $a \in \mathcal{P}, R$ is welldefined for $f \in l^{1}\left(\mathbf{Z}^{n}\right)$ and

$$
\sum_{k \in \mathbf{Z}}|R f(H(a, k))| \leq\|f\|_{1} .
$$

The slice formula for the discrete Radon transform $R$ is given as follows. For $f \in l^{1}\left(\mathbf{Z}^{n}\right)$ and $\varphi \in l^{\infty}(\mathbf{Z})$,

$$
\begin{aligned}
\sum_{k \in \mathbf{Z}} R f(H(a, k)) \varphi(k) & =\sum_{k \in \mathbf{Z}}\left(\sum_{m \in H(a, k)} f(m)\right) \varphi(k) \\
& =\sum_{m \in \mathbf{Z}^{n}} f(m) \varphi(a m) .
\end{aligned}
$$

Especially, letting $\varphi_{\lambda}(k)=e^{i \lambda k}, 0 \leq \lambda<2 \pi$, we see that

$$
\widetilde{R f}(H(a, \cdot))(\lambda)=\widetilde{f}(\lambda a),
$$

where the tildes denote the Fourier inverse transforms on $\mathbf{Z}$ and $\mathbf{Z}^{n}$, that is, the Fourier series on $\mathbf{T}$ and $\mathbf{T}^{n}$ respectively.

The inversion formula for $R$ is given as follows. Let $\chi_{N}$ be the characteristic function of a discrete ball $B(N)=\left\{x \in \mathbf{Z}^{n} \mid\|x\| \leq N\right\}$, where $\|\cdot\|$ denotes the Euclidean norm on $\mathbf{R}^{n}$. Then, for all $\varepsilon>0$, there exists a sufficiently large $N$ for which $\left\|f-f \chi_{N}\right\|_{1}<\varepsilon$ and thus, by (2)

$$
\left|R\left(f \chi_{N}\right)(H(a, k))-R f(H(a, k))\right|<\varepsilon
$$

for all $a \in \mathcal{P}$. Let $a_{j}=\left(1, j, j^{2}, \ldots, j^{n-1}\right)$ for $j \in \mathbf{N}$. As shown in [1], if $j>N$, then $B(N) \cap H\left(a_{j}, 0\right)=\{0\}$ and thus, $R\left(f \chi_{N}\right)(H(a, 0))=f \chi_{N}(0)=f(0)$. Therefore, combining the above inequality, we have the following inversion formula: For $f \in l^{1}\left(\mathbf{Z}^{n}\right)$,

$$
\lim _{j \rightarrow \infty} R f\left(a_{j}, 0\right)=f(0) .
$$

When $n=2$, we can prove that $B(N) \cap H(a, 0)=\{0\}$ if $\|a\|>N^{\dagger}$. Hence, it follows that for $f \in l^{1}\left(\mathbf{Z}^{2}\right)$,

$$
\lim _{\|a\| \rightarrow \infty} R f(a, 0)=f(0) .
$$

\section{Schwartz space}

Let $\mathcal{S}\left(\mathbf{Z}^{n}\right)$ be the Schwartz space on $\mathbf{Z}^{n}$ consisting of all functions $f$ on $\mathbf{Z}^{n}$ such that

$$
p_{N}(f)=\sup _{m \in \mathbf{Z}^{n}}\left(1+\|m\|^{2}\right)^{N}|f(m)|<\infty
$$

\footnotetext{
${ }^{\dagger}$ This is not true when $n>2$. For example, let $n=3$ and $a_{p}=(p, 2,-(p+2))$ for a prime number $p$. Then $(1,1,1) \in H\left(a_{p}, 0\right)$ for all $a_{p}$.
} 
for all $N=0,1,2, \ldots$ Then $\left\{p_{N} \mid N=0,1,2, \ldots\right\}$ is a family of semi-norms of $\mathcal{S}\left(\mathbf{Z}^{n}\right)$. We note that, for $f \in \mathcal{S}\left(\mathbf{Z}^{n}\right)$ and $N \in \mathbf{N}$, if $2 N>n$, then

$$
\begin{aligned}
& \left(1+k^{2}\right)^{N} R f(H(a, k)) \\
\leq & \sum_{\{m \mid a m=k\}}|f(m)|\left(1+|a m|^{2}\right)^{N} \\
\leq & \sum_{\{m \mid a m=k\}}|f(m)|\left(1+\|a\|^{2}\right)^{N}\left(1+\|m\|^{2}\right)^{N} \\
\leq & p_{2 N}(f) \sum_{m \in \mathbf{Z}^{n}}\left(1+\|m\|^{2}\right)^{-N} \cdot\left(1+\|a\|^{2}\right)^{N} .
\end{aligned}
$$

Therefore, it follows that

$$
|R f(H(a, k))| \leq c_{N} p_{2 N}(f)\left(\frac{1+\|a\|^{2}}{1+k^{2}}\right)^{N},
$$

where $c_{N}$ is independent of $a$ and $k$. In what follows, for simplicity, we assume that $n=2$. In the case of general $n$, the same arguments are easily applicable. For $a=\left(a_{1}, a_{2}\right) \in \mathcal{P}$, $H(a, k), k \in \mathbf{Z}$, are discrete hyperplanes with the same direction and they cover $\mathbf{Z}^{2}$. For each $k \in \mathbf{Z}$, we choose $m \in H(a, k)$ that is nearest to the origin. We denote it by $m_{0}(a, k)$ and set

$$
D(a)=\left\{m_{0}(a, k) \mid k \in \mathbf{Z}\right\},
$$

where we take $m_{0}(a, 0)=0$. Clearly, we see that

$$
H(a, k)=\left\{m_{0}+l a_{0} \mid l \in \mathbf{Z}\right\},
$$

where $a_{0}=\left(-a_{2}, a_{1}\right)$ and $m_{0}=m_{0}(a, k) \in D(a)$. Then it follows that

$$
\begin{aligned}
& R f(H(a, k))=\sum_{m \in H(a, k)} f(m) \\
& =f\left(m_{0}\right)+\sum_{0<|l| \leq 4} f\left(m_{0}+l a_{0}\right)+\sum_{|l|>4 \frac{\left\|m_{0}\right\|}{\|a\|}} f\left(m_{0}+l a_{0}\right) \\
& =f\left(m_{0}\right)+I_{1}+I_{2} \text {. }
\end{aligned}
$$

As for $I_{1}$, since $|f(m)| \leq p_{N}(f)\left(1+\|m\|^{2}\right)^{-N} \leq p_{N}(f)\left(1+\left\|m_{0}\right\|^{2}\right)^{-N}$,

$$
\begin{aligned}
\left|I_{1}\right| & \leq p_{N}(f)\left(1+\left\|m_{0}\right\|^{2}\right)^{-N} 4 \frac{\left\|m_{0}\right\|}{\|a\|} \\
& \leq \frac{c}{\|a\|\left(1+\left\|m_{0}\right\|\right)^{2 N-1}},
\end{aligned}
$$


where $c$ is independent of $a$ and $k$. As for $I_{2}$, we note that $\left\|a_{0}\right\|=\|a\|$ and $2 \operatorname{lm}_{0} a_{0} \geq$ $-2|l|\left\|m_{0}\right\|\left\|a_{0}\right\| \geq-\frac{|l|^{2}\|a\|^{2}}{2}$. Hence

$$
\|m\|^{2}=\left\|m_{0}\right\|^{2}+l^{2}\|a\|^{2}+2 l m_{0} a \geq\left\|m_{0}\right\|^{2}+\frac{l^{2}\|a\|^{2}}{2}
$$

and thus,

$$
|f(m)| \leq p_{N}(f)\left(1+\left\|m_{0}\right\|^{2}+\frac{l^{2}\|a\|^{2}}{2}\right)^{-N} .
$$

Therefore, if $N>1$, then

$$
\begin{aligned}
\left|I_{2}\right| & \leq c p_{N}(f) \sum_{|l|>4 \frac{\left\|m_{0}\right\|}{\|a\|}}\left(1+\left\|m_{0}\right\|^{2}+\frac{l^{2}\|a\|^{2}}{2}\right)^{-N} \\
& \leq 2 c p_{N}(f) \int_{4 \frac{\left\|m_{0}\right\|}{\|a\|}}^{\infty}\left(1+\left\|m_{0}\right\|^{2}+\frac{x^{2}\|a\|^{2}}{2}\right)^{-N} d x \\
& \leq \frac{c}{\|a\|\left(1+\left\|m_{0}\right\|\right)^{2 N-1}},
\end{aligned}
$$

where $c$ is independent of $a$ and $k$. Hence we can deduce that $R f(H(a, k))$ has a decomposition

$$
R f(H(a, k))=f\left(m_{0}\right)+g(a, k)
$$

and for each $N=0,1,2, \ldots$,

$$
g(a, k) \leq \frac{c}{\|a\|\left(1+\left\|m_{0}\right\|\right)^{N}},
$$

where $c$ is independent of $a$ and $k$. Moreover, noting (3) and (4), we see that

$$
\begin{aligned}
\widetilde{R f}(H(a, \cdot))(\lambda) & =\sum_{m \in \mathbf{Z}^{n}} f(m) e^{i \lambda a m} \\
& =\sum_{m_{0} \in D(a)} f\left(m_{0}\right) e^{i \lambda a m_{0}}+\sum_{m \in D(a)^{c}} f(m) e^{i \lambda a m} \\
& =\widetilde{\left.f\right|_{D(a)}}(\lambda a)+\widetilde{\left.f\right|_{D(a)^{c}}}(\lambda a) .
\end{aligned}
$$

On the other hand, from (7) we see that

$$
\begin{aligned}
\widetilde{R f}(H(a, \cdot))(\lambda) & =\sum_{k \in \mathbf{Z}}\left(f\left(m_{0}(a, k)\right) e^{i \lambda k}+g(a, k) e^{i \lambda k}\right) \\
& =\widetilde{\left.f\right|_{D(a)}}(\lambda a)+\widetilde{g}(a, \cdot)(\lambda) .
\end{aligned}
$$

Hence we can obtain that

$$
\widetilde{g}(a, \cdot)(\lambda)=\widetilde{\left.f\right|_{D(a)^{c}}}(\lambda a) .
$$


We now consider a characterization of the image of the discrete Radon transforms $R f(H(a, k))$ of $f \in \mathcal{S}\left(\mathbf{Z}^{2}\right)$.

Proposition 3.1. Let $F(a, k)$ and $f(m)$ be functions on $\mathbf{G}$ and $\mathbf{Z}^{2}$ respectively. We suppose that

$$
F(a, k)=f\left(m_{0}\right)+g(a, k),
$$

where $m_{0}=m(a, k) \in D(a)$ and $g(a, k)$ is a function on $\mathbf{G}$, which satisfies that for each $N=0,1,2, \ldots$,

$$
g(a, k) \leq \frac{c}{\|a\|\left(1+\left\|m_{0}\right\|\right)^{N}},
$$

where $c$ is independent of $a$ and $k$. Then

$$
\lim _{\|a\| \rightarrow \infty} F(a, a m)=f(m)
$$

for all $m \in \mathbf{Z}^{2}$. Especially, the above decomposition of $F$ is unique. Furthermore, $F$ satisfies that for each $N=0,1,2, \ldots$,

$$
|F(a, k)| \leq c\left(\frac{1+\|a\|^{2}}{1+k^{2}}\right)^{N},
$$

where $c$ is independent of $a$ and $k$, if and only if $f \in \mathcal{S}\left(\mathbf{Z}^{2}\right)$.

Proof. We fix $m \in \mathbf{Z}^{2}$ and consider $a \in \mathcal{P}$ such that $\|a\|>2\|m\|^{\ddagger}$. Then it easily follows that

$$
m_{0}(a, a m)=m
$$

and thus,

$$
|F(a, a m)-f(m)| \leq \frac{c}{\|a\|(1+\|m\|)^{N}},
$$

where $c$ is independent of $a$ and $m$, Therefore,

$$
\lim _{\|a\| \rightarrow \infty} F(a, a m)=f(m) .
$$

We suppose that $F(a, k)$ satisfies (11). Without loss of generality, we may suppose that, for $m=\left(m_{1}, m_{2}\right), m_{2} \neq 0$ and $\left|m_{2}\right| \geq\left|m_{1}\right|$. Let $a_{j}=(1, j) \in \mathcal{P}$ and $\left\|a_{j}\right\|>2\|m\|$. Then for each $N=0,1,2, \ldots$,

$$
\begin{aligned}
\left(1+\|m\|^{2}\right)^{N}\left|F\left(a_{j}, a_{j} m\right)\right| & \leq c\left(1+\|m\|^{2}\right)^{N}\left(\frac{1+\left\|a_{j}\right\|^{2}}{1+\left(a_{j} m\right)^{2}}\right)^{N} \\
& \leq c\left(1+m_{1}^{2}+m_{2}^{2}\right)^{N}\left(\frac{1+1+j^{2}}{1+\left(m_{1}+j m_{2}\right)^{2}}\right)^{N}
\end{aligned}
$$

\footnotetext{
\$) See REMARK 3.2.
} 


$$
\begin{aligned}
& \leq c\left(1+m_{2}^{2}\right)^{N}\left(\frac{1+j^{2}}{1+(j-1)^{2} m_{2}^{2}}\right)^{N} \\
& \leq c,
\end{aligned}
$$

where $c$ is independent of $a_{j}$ and $m$. Hence, by multiplying $\left(1+\|m\|^{2}\right)^{N}$ to the both sides of (12) replaced $a$ and $N$ by $a_{j}$ and $2 N$ respectively, and then, by letting $j$ go to $\infty$, it follows that

$$
\sup _{m \in \mathbf{Z}^{2}}\left(1+\|m\|^{2}\right)^{N}|f(m)|<\infty .
$$

Conversely, we suppose that $f \in \mathcal{S}\left(\mathbf{Z}^{n}\right)$. Since $m_{0}=m_{0}(a, k)$ is in $H(a, k)$ and thus, lies on the line $m_{0}+t a_{0}, t \in \mathbf{R}$, and $a \perp a_{0}$. Hence

$$
\left\|m_{0}\right\| \geq \frac{\left|m_{0} a\right|}{\|a\|}=\frac{|k|}{\|a\|}
$$

and thus,

$$
\frac{1}{1+\left\|m_{0}\right\|^{2}} \leq \frac{1+\|a\|^{2}}{1+k^{2}} .
$$

Therefore, the desired result follows from the decomposition of $F$.

REMARK 3.2. As pointed in $\S 2$, when $n>2$, the inversion formula (10) in Proposition 3.1 is not true. The one replaced $a$ by $a_{j}$ holds.

Proposition 3.3. Let $F(a, k)=f\left(m_{0}\right)+g(a, k)$, where $m_{0}=m(a, k) \in D(a)$. We suppose that $f$ belongs to $\mathcal{S}\left(\mathbf{Z}^{2}\right)$ and $\widetilde{g}(a, \cdot)(\lambda)=\widetilde{\left.f\right|_{D(a)^{c}}}(\lambda a)$. Then for each $N=$ $0,1,2, \ldots$,

$$
|g(a, k)| \leq \frac{c}{\|a\|\left(1+\left\|m_{0}\right\|\right)^{N}},
$$

where $c$ is independent of $a$ and $k$.

Proof. We note that

$$
\begin{aligned}
g(a, k) & =\int_{\mathbf{T}} \widetilde{\left.f\right|_{D(a)^{c}}(\lambda a) e^{-i \lambda k} d \lambda} \\
& =\sum_{m \in D(a)^{c}, m a=k} f(m) \\
& =\sum_{m \neq m_{0}(a, k) \in H(a, k)} f(m) .
\end{aligned}
$$

As in the calculation that yields (8), $g$ satisfies the desired estimate.

We now define $\mathcal{S}_{*}(\mathbf{G})$ as follows. 
DEFinition 3.4. Let $\mathcal{S}_{*}(\mathbf{G})$ be the space of all $F(a, k)$ on $\mathbf{G}$ being of the form

$$
F(a, k)=f\left(m_{0}\right)+g(a, k),
$$

where $f \in \mathcal{S}\left(\mathbf{Z}^{2}\right), m_{0}=m_{0}(a, k) \in D(a)$ and $g$ satisfies that for $\lambda \in \mathbf{T}$,

$$
\tilde{g}(a, \cdot)(\lambda)=\widetilde{\left.f\right|_{D(a)^{c}}}(\lambda a) .
$$

According to Propositions 3.1 and 3.3, if $F \in \mathcal{S}_{*}(\mathbf{G})$, then the decomposition $F(a, k)=$ $f\left(m_{0}\right)+g(a, k)$ is unique and the following properties hold: For each $N=0,1,2, \ldots$,

$$
\begin{gathered}
|g(a, k)| \leq \frac{c}{\|a\|\left(1+\left\|m_{0}\right\|\right)^{N}}, \\
|F(a, k)| \leq c\left(\frac{1+\|a\|^{2}}{1+k^{2}}\right)^{N}, \\
\lim _{\|a\| \rightarrow \infty} F(a, a m)=f(m)^{\ddagger},
\end{gathered}
$$

where $c$ is independent of $a$ and $k$. We define for all $N=0,1,2, \ldots$,

$$
q_{N}(F)=\sup _{a \in \mathcal{P}, k \in \mathbf{Z}}\left(\frac{1+\|a\|^{2}}{1+k^{2}}\right)^{-N}|F(a, k)| .
$$

Then $\left\{q_{N} \mid N=0,1,2, \ldots\right\}$ is a family of semi-norms of $\mathcal{S}_{*}(\mathbf{G})$.

Our main theorem is the following.

THEOREM 3.5. $R$ is a bijective continuous map from $S\left(\mathbf{Z}^{2}\right)$ to $S_{*}(\mathbf{G})$.

Proof. From (6), (7), (8), (9), and Propositions 3.1, it follows that $R$ is an injective continuous map from $S\left(\mathbf{Z}^{n}\right)$ to $S_{*}(\mathbf{G})$. We shall prove that $R$ is surjective. Let $F$ be in $S_{*}(\mathbf{G})$ and $F=f+g$ denote the decomposition (13) of $F$ in Definition 3.4. Since $f \in S\left(\mathbf{Z}^{n}\right), R f \in$ $S_{*}(\mathbf{G})$ and thus, $H=F-R f$ belongs to $S_{*}(\mathbf{G})$. By noting (7), the unique decomposition (13) of $H$ is of the form $H=0+g^{\prime}$. Hence $\widetilde{g^{\prime}}(a, \cdot)=0$ for all $a \in \mathcal{P}$ by (14). Then $g^{\prime}=0$ and thus, $F=R g$.

REMARK 3.6. The relation (14) in Definition 3.4 is used to prove that, if $f=0$, then $F(a, \cdot)=0$ for all $a \in \mathcal{P}$. Since $R f(H(a, k))$ satisfies (3), we may replace the relation by the following condition: Let $\mathcal{H}$ be an infinite dimensional Hilbert space and $\left\{v_{k} \mid k \in \mathbf{Z}\right\}$ a complete orthonormal system of $\mathcal{H}$. Then $F$ and $f$ satisfy

$$
\sum_{k \in \mathbf{Z}} F(a, k) v_{k}=\sum_{m \in \mathbf{Z}^{n}} f(m) v_{a m}
$$

for all $a \in \mathcal{P}$. Actually, if $f=0$, then $F(a, \cdot)=0$ for all $a \in \mathcal{P}$.

\footnotetext{
${ }^{\ddagger}$ See REMARK 3.2 .
} 


\section{Inversion formula}

In the following, let $n=2$. In addition to (5) we shall obtain several methods of recovering $f$ from $R f$. For $a, b \in \mathbf{N}$, let $[a, b]$ denote the sets of integers $p$ such that $a \leq p \leq b$. For a set $S \subset \mathbf{Z}^{2}$, let $\chi_{S}$ denote the characteristic function of $S$ and $|S|$ the cardinality of $S$.

4.1. Mean inversion formula. Let $Q$ be a direction function on $\mathbf{Z}^{2}$ which depends only on directions:

$$
Q(0)=0, \quad Q(x)=Q(a)=Q(-a) \text { for } x=l a,
$$

where $a \in \mathcal{P}$ and $l \neq 0 \in \mathbf{Z}$. Suppose that $\|Q\|_{\infty}<\infty$ and $\sum_{a \in \mathcal{P}} Q(a)<\infty$. Then for $f \in l^{1}\left(\mathbf{Z}^{2}\right)$, since $Q(0)=0$, it follows that

$$
\begin{aligned}
Q * f(m) & =\sum_{m^{\prime} \in \mathbf{Z}^{2}} Q\left(m-m^{\prime}\right) f\left(m^{\prime}\right) \\
& =\frac{1}{2} \sum_{a \in \mathcal{P}} \sum_{l \neq 0 \in \mathbf{Z}} Q(a) f(m+l a) \\
& =\frac{1}{2} \sum_{a \in \mathcal{P}} Q\left(a_{0}\right) R f(H(a, a m))-\frac{1}{2} f(m) \sum_{a \in \mathcal{P}} Q(a),
\end{aligned}
$$

where $a \perp a_{0}$ and $a_{0} \in \mathcal{P}$. Hence we can obtain the following.

THEOREM 4.1. Let $Q$ be a direction function on $\mathbf{Z}^{2}$ and suppose that $\|Q\|_{\infty}<\infty$ and $\sum_{a \in \mathcal{P}} Q(a) \neq 0<\infty$. Then for $f \in l^{1}\left(\mathbf{Z}^{2}\right)$,

$$
f(m)=\frac{1}{\sum_{a \in \mathcal{P}} Q(a)}\left(\sum_{a \in \mathcal{P}} Q\left(a_{0}\right) R f(H(a, a m))-2 Q * f(m)\right) .
$$

COROllary 4.2. Let $\left\{Q_{i}\right\}, i \in \mathbf{N}$, be a sequence of direction functions on $\mathbf{Z}^{2}$ satisfying

$$
\begin{aligned}
& \text { (a) }\left\|Q_{i}\right\|_{\infty}<C \text { for all } i, \\
& \text { (b) } \sum_{a \in \mathcal{P}} Q_{i}(a) \rightarrow \infty \text { if } i \rightarrow \infty
\end{aligned}
$$

Then for $f \in l^{1}\left(\mathbf{Z}^{2}\right)$,

$$
f(m)=\lim _{i \rightarrow \infty} \frac{1}{\sum_{a \in \mathcal{P}} Q_{i}(a)} \sum_{a \in \mathcal{P}} Q_{i}\left(a_{0}\right) R f(H(a, a m)) .
$$

For example, if we take $Q_{i}(x)=\chi_{B(i)}(a)$ for $x=l a$, then we see that

$$
f(m)=\lim _{i \rightarrow \infty} \frac{1}{|B(i) \cap \mathcal{P}|} \sum_{a \in B(i) \cap \mathcal{P}} R f(H(a, a m)) .
$$


Proof. Since $\left\|Q_{i} * f\right\|_{\infty} \leq\left\|Q_{i}\right\|_{\infty}\|f\|_{1} \leq C\|f\|_{1},\left(\sum_{a \in \mathcal{P}} Q_{i}(a)\right)^{-1} Q_{i} * f \rightarrow 0$ if $i \rightarrow \infty$ by $(b)$. Hence the desired formula follows from (16).

COROllary 4.3. Let $\left\{Q_{i}\right\}, i \in \mathbf{N}$, be a sequence of direction functions on $\mathbf{Z}^{2}$. Furthermore, we suppose that

$$
\begin{aligned}
& \text { (a) }\left\|Q_{i}\right\|_{\infty}<C \text { for all } i, \\
& \text { (b) } \operatorname{supp} Q_{i} \subset B\left(r_{i}\right)^{c} \text { where } r_{i} \rightarrow \infty \text { if } i \rightarrow \infty, \\
& \text { (c) } \lim _{i \rightarrow \infty} \sum_{a \in \mathcal{P}} Q_{i}(a)>0 .
\end{aligned}
$$

Then for $f \in l^{1}\left(\mathbf{Z}^{2}\right)$,

$$
f(m)=\lim _{i \rightarrow \infty} \frac{1}{\sum_{a \in \mathcal{P}} Q_{i}(a)} \sum_{a \in \mathcal{P}} Q_{i}\left(a_{0}\right) R f(H(a, a m)) .
$$

For example, if we take a finite subset $S_{i}$ in $B\left(r_{i}\right)^{c}$ and let $Q_{i}(x)=\chi_{S_{i}}(a)$ for $x=$ la, then

$$
f(m)=\lim _{i \rightarrow \infty} \frac{1}{\left|S_{i}\right|} \sum_{a \in S_{i}} R f(H(a, a m)) .
$$

Proof. Since $Q_{i}(0)=0,\left|Q_{i} * f(m)\right| \leq\left\|Q_{i}\right\|_{\infty} \sum_{m^{\prime} \in B\left(r_{i}\right)^{c}}\left|f\left(m+m^{\prime}\right)\right| \leq$ $C \sum_{m^{\prime} \in B\left(r_{i}\right)^{c}}\left|f\left(m+m^{\prime}\right)\right|$. Hence, $\left|Q_{i} * f(m)\right| \rightarrow 0$ if $i \rightarrow \infty$, because $f \in l^{1}\left(\mathbf{Z}^{2}\right)$ and (b). Therefore, the desired formula follows from (16) and (c).

REMARK 4.4. These corollaries are generalizations of the formulas obtained in [2], Theorem 2.1 (a) and (b). In Corollary 4.3, if we take $S_{i}=\left\{a_{i}\right\}, a_{i} \in \mathcal{P}$, then we can deduce (5).

4.2. Discrete Fourier inversion formula. We introduce an inversion formula using the discrete Fourier transform.

Step1. We first suppose that supp $f \subset[-N, N]^{2}$. Since $|a m| \leq\left(\left|a_{1}\right|+\left|a_{2}\right|\right) N=|a| N$, where $|a|=\left|a_{1}\right|+\left|a_{2}\right|$, for $a=\left(a_{1}, a_{2}\right) \in \mathcal{P}$ and $m \in[-N, N]^{2}$, the support of $R f(H(a, k))$ with respect to $k$ is in $[-|a| N,|a| N]$. We recall the discrete Fourier transform on $[-N, N]^{2}$ and its inversion formula: For $t=\left(t_{1}, t_{2}\right), 0 \leq t_{1}, t_{2} \leq 2 N$, the discrete Fourier transform $F(t)$ of $f(n)$ is given by

$$
F(t)=\frac{1}{(2 N+1)^{2}} \sum_{n_{1}, n_{2}=0}^{2 N} f\left(\left(n_{1}, n_{2}\right)-(N, N)\right) e^{-i \frac{2\left(n_{1} t_{1}+n_{2} t_{2}\right) \pi}{2 N+1}}
$$


and for $n=\left(n_{1}, n_{2}\right),-N \leq n_{1}, n_{2} \leq N, f(m)$ is recovered as

$$
\begin{aligned}
f(n) & =\sum_{t_{1}, t_{2}=0}^{2 N} F(t) e^{i \frac{2\left(\left(n_{1}+N\right) t_{1}+\left(n_{2}+N\right) t_{2}\right) \pi}{2 N+1}} \\
& =\sum_{t \in[0,2 N]^{2}} F(t)(-1)^{|t|} e^{i \frac{(2 n t-|t|) \pi}{2 N+1}} .
\end{aligned}
$$

Step2. We apply the slice formula (3). For each $a=\left(a_{1}, a_{2}\right) \in \mathcal{P}$ with $a_{1}, a_{2} \geq 0$, and $l \in \mathbf{Z}$,

$$
\begin{aligned}
& \sum_{k=-|a| N}^{|a| N} R f(H(a, k)) e^{-i \frac{2(k+|a| N) l \pi}{2 N+1}} \\
= & \sum_{m \in \mathbf{Z}^{2}} f(m) e^{-i \frac{2(a m+|a| N) l \pi}{2 N+1}} \\
= & \sum_{m \in \mathbf{Z}^{2}} f\left(\left(m_{1}, m_{2}\right)-(N, N)\right) e^{-i \frac{2 l\left(m_{1} a_{1}+m_{2} a_{2}\right) \pi}{2 N+1}} \\
= & (2 N+1)^{2} F(l a) .
\end{aligned}
$$

Step3. We combine (17) and (18). Let $\mathcal{P}(N)=\mathcal{P} \cap[0,2 N]^{2}$ and $\mathbf{Z}_{+}$the set of the positive integers. We denote $t=\left(t_{1}, t_{2}\right) \neq(0,0), 0 \leq t_{1}, t_{2} \leq 2 N$, as

$$
t=\left(t_{1}, t_{2}\right)=l_{t} a_{t},
$$

where $a_{t} \in \mathcal{P}(N)$ and $l_{t} \in \mathbf{Z}_{+}$. When $t=(0,0)$, we let $l_{t}=0$ and $a_{t}$ is arbitrary. Then, replacing $F(t)=F\left(l_{t} a_{t}\right)$ in (17) with (18), we see that

$$
\begin{aligned}
f(n)= & \frac{1}{(2 N+1)^{2}} \sum_{t \in[0,2 N]^{2}}\left(\sum_{k=-\left|a_{t}\right| N}^{\left|a_{t}\right| N} R f\left(H\left(a_{t}, k\right)\right) e^{-i \frac{2\left(k+\left|a_{t}\right| N\right) l_{t} \pi}{2 N+1}}\right) \\
& \times(-1)^{|t|} e^{i \frac{(2 n t-|t| \pi}{2 N+1}} \\
= & \frac{1}{(2 N+1)^{2}} \sum_{t \in[0,2 N]^{2}}\left(\sum_{k=-\left|a_{t}\right| N}^{\left|a_{t}\right| N} R f\left(H\left(a_{t}, k\right)\right) e^{-i \frac{2\left(k-n a_{t}\right) l_{t} \pi}{2 N+1}}\right) .
\end{aligned}
$$

For $a \in \mathcal{P}(N)$ let $L(a, N)=\max \left\{l \in \mathbf{N} \mid l a \in[0,2 N]^{2}\right\}$. We recall that, when $t=(0,0)$, $l_{t}=0$, and that $\sum_{k \in \mathbf{Z}} R f\left(H\left(a_{t}, k\right)\right)=\sum_{m \in \mathbf{Z}^{2}} f(m)$. Hence, we can rewrite the previous equation as

$$
f(n)=\frac{1}{(2 N+1)^{2}}\left(\sum_{a \in \mathcal{P}(N)}\left(\sum_{k=-|a| N}^{|a| N} R f(H(a, k)) \sum_{l=1}^{L(a, N)} e^{-i \frac{2(k-n a) l \pi}{2 N+1}}\right)\right) .
$$


Step4. Let $f$ be an arbitrary function in $l^{1}\left(\mathbf{Z}^{2}\right)$. Then it is easy to see that

$$
\begin{aligned}
& \frac{1}{(2 N+1)^{2}}\left|\sum_{a \in \mathcal{P}(N)}\left(\sum_{k=-|a| N}^{|a| N} R\left(f-f \chi_{N}\right)(H(a, k)) \sum_{l=1}^{L(a, N)} e^{-i \frac{2(k-n a) l \pi}{2 N+1}}\right)\right| \\
\leq & \frac{1}{(2 N+1)^{2}} \sum_{a \in \mathcal{P}(N)} L(a, N) \sum_{k=-|a| N}^{|a| N}\left|R\left(f-f \chi_{N}\right)(H(a, k))\right| \\
\leq & \frac{N^{2}}{(2 N+1)^{2}} \sum_{\|m\|>N}|f(m)| .
\end{aligned}
$$

Since the last term goes to 0 if $N \rightarrow \infty$, we can obtain the following.

THEOREM 4.5. For each $N \in \mathbf{N}$, let $\mathcal{P}(N)=\mathcal{P} \cap[0,2 N]^{2}$ and for each $a \in \mathcal{P}(N)$, let $L(a, N)=\max \left\{l \in \mathbf{N} \mid l a \in[0,2 N]^{2}\right\}$. Then for $f \in l^{1}\left(\mathbf{Z}^{2}\right)$,

$$
f(n)=\lim _{N \rightarrow \infty} \frac{1}{(2 N+1)^{2}} \sum_{a \in \mathcal{P}(N)}\left(\sum_{k=-|a| N}^{|a| N} R f(H(a, k)) \sum_{l=1}^{L(a, N)} e^{-i \frac{2(k-n a) l \pi}{2 N+1}}\right) .
$$

4.3. Algorithmic inversion formula. We introduce a method to recover $f(0,0)$ from $R f(H(a, k))$ by an algorithmic process. We first note that, if $f$ is supported on $[-N, N]^{2}$ and $\|a\|>N$, then

$$
R f(H(a, 0))=f(0,0),
$$

because $[-N, N]^{2} \cap H(a, 0)=\{(0,0)\}$ (see Fig. 1).

In the following, we shall consider an algorithm by which $f(0,0)$ is recovered from $R f(H(a, k))$ with $\|a\| \leq N$.

Step1. For each $j=0,1,2, \ldots$, we first define a set $V(N, j)$ of points in $[-N, N]^{2}$ and a set $E(N, j)$ of hyperplanes on $\mathbf{Z}^{2}$ inductively. The case of $N=3$ is referred to Example

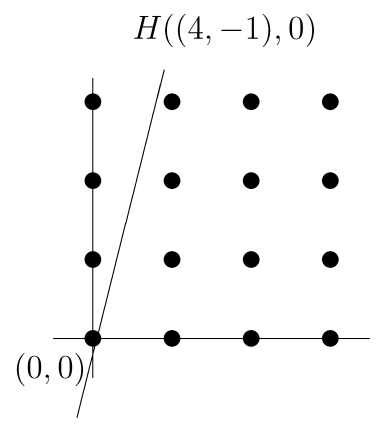

Figure 1. $\quad N=3$ and $a=(4,-1)$ 
4.6. Let

$$
E(N, 0)=\emptyset, V(N, 0)=[-N, N]^{2} .
$$

Let $E(N, 1)$ be the set of hyperplanes $H(a, a p)$ through $p \in V(N, 0)$ such that

(i) $H(a, a p) \cap V(N, 0)=\{p\}$

(ii) $\|a\|$ is minimum among $H(a, a p)$ satisfying $(i)$

and $V(N, 1)=V(N, 0)-\{p \mid H(a, a p) \in E(N, 1)\}$. Furthermore, inductively, we define $E(N, j+1)$ as the set of hyperplanes $H(a, a p)$ through $p \in V(N, j)$ such that

(i) $H(a, a p) \cap V(N, j)=\{p\}$

(ii) $\|a\|$ is minimum among $H(a, a p)$ satisfying $(i)$

and $V(N, j+1)=V(N, j)-\{p \mid H(a, a p) \in E(N, j+1)\}$.

We note that there exists $j_{N}$ for which $V\left(N, j_{N}-1\right)$ is not contained in [- $(N-1), N-$ $1]^{2}$, but

$$
V\left(N, j_{N}\right) \subset[-(N-1), N-1]^{2} .
$$

EXAMPLE 4.6. Let $N=3$. In the following figures we denote the area only in the first quadrant.

$$
\begin{aligned}
& V(3, j)_{+}=V(3, j) \cap[0,3]^{2}, \\
& E(3, j)_{+}=\left\{H(a, a p) \in E(3, j) \mid p \in[0,3]^{2}\right\} .
\end{aligned}
$$

In the first line of Fig. 2, we let $a=(1,1)$ and $p=(3,3)$. Then $H(a, 6) \cap V(3,0)=\{p\}$ and $\|a\|=\sqrt{2}$. Hence it follows that

$$
\begin{aligned}
& E(3,1)_{+}=\{H((1,1), 6)\} \\
& V(3,1)_{+}=[0,3]^{2}-\{(3,3)\} .
\end{aligned}
$$

In the second line in Fig. 2 , we let $a=(1,2),(2,1)$ and $p=(2,4),(4,2)$ respectively. Then $H(a, 10) \cap V(3,1)=\{p\}$ and $\|a\|=\sqrt{5}$. Hence it follows that

$$
\begin{aligned}
& E(3,2)_{+}=\{H((1,2), 10), H((2,1), 10)\} \\
& V(3,2)_{+}=V(3,1)_{+}-\{(2,4),(4,2)\} .
\end{aligned}
$$

Finally, we see that $V(3,4)+\subset[0,2]^{2}$ and thus, $j_{3}=4$.

Step2. We define an operator Shave ${ }_{N}$ for a function $f$ on $[-N, N]^{2}$. We note that, if $f$ is supported in $V(N, j)$, then $R f(H(a, a p))=f(p)$ for each $H(a, a p) \in E(N, j)$. We define a set of functions $\left\{f_{j}\right\}$ inductively as 

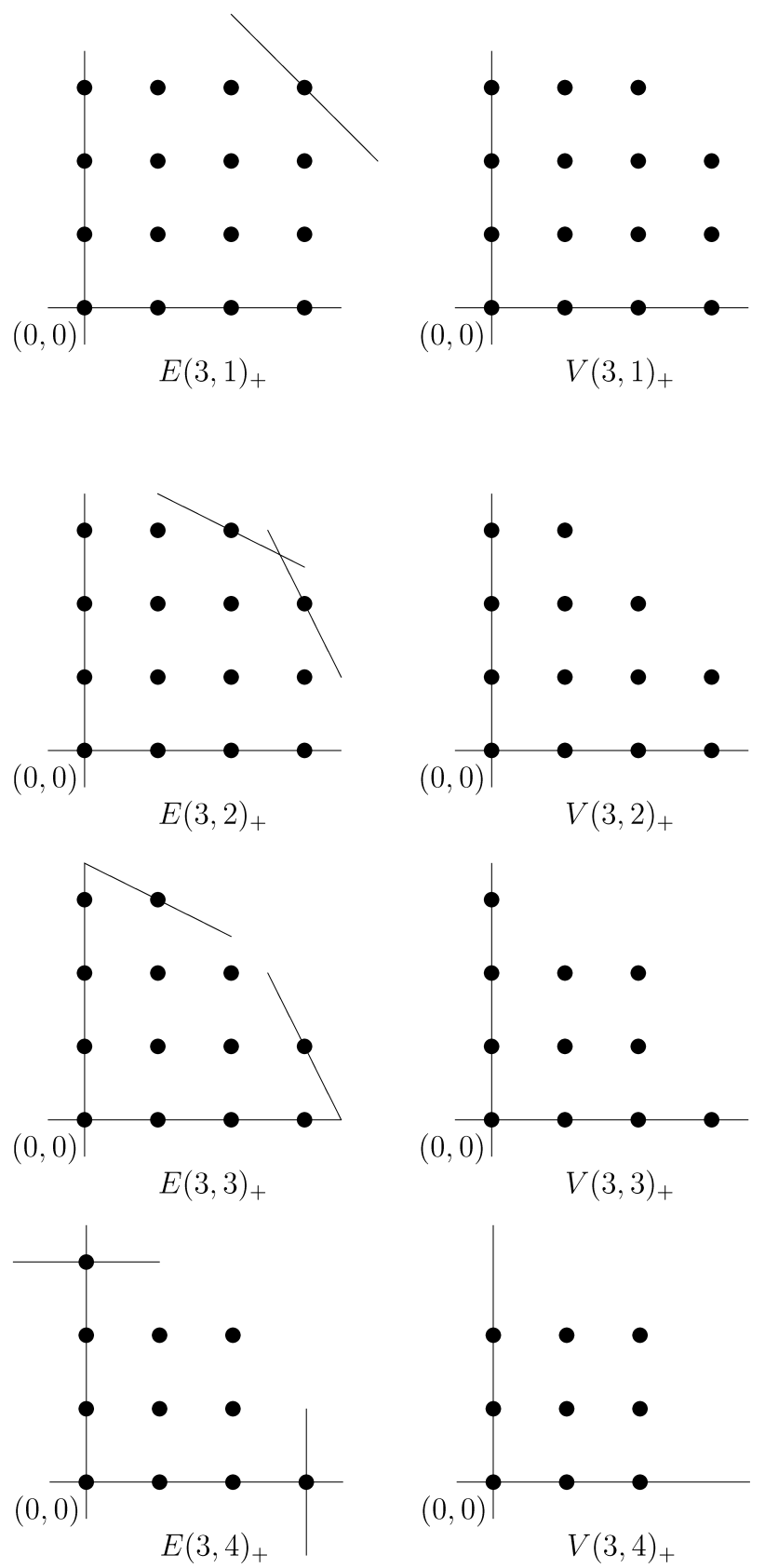

FIGURE 2. 


$$
\begin{gathered}
f_{1}=f-\sum_{H(a, a p) \in E(N, 1)} R f(H(a, a p)) \delta_{p}, \\
f_{j+1}=f_{j}-\sum_{H(a, a p) \in E(N, j+1)} R f_{j}(H(a, a p)) \delta_{p}
\end{gathered}
$$

and put

$$
\operatorname{Shave}_{N}(f)=f_{j_{N}} \text {. }
$$

Clearly, Shave $_{N}(f)$ is supported on $[-(N-1), N-1]^{2}$.

Step3. We replace $N$ by $N-1$ and apply the previous arguments to $\operatorname{Shave}_{N}(f)$, which is supported on $[-(N-1), N-1]^{2}$. Furthermore, we repeat the process successively. Then we can easily deduce that

$$
\begin{aligned}
f(0,0) \delta_{(0,0)} & =\text { Shave }_{1} \circ \text { Shave }_{2} \circ \cdots \circ \text { Shave }_{N-1} \circ \text { Shave }_{N}(f) \\
& =f-\sum_{\substack{p \in[-N, N]^{2}, p \neq(0,0)}} d_{N}(R f, p) \delta_{p},
\end{aligned}
$$

where $d_{N}(R f, p)$ is a linear combination of $R f(H)$ with

$$
H \in \cup_{n=1}^{N} \bigcup_{j=1}^{j_{n}} E(n, j)
$$

Therefore, for $p \neq(0,0) \in[-N, N]^{2}$, it follows that

$$
f(p)=d_{N}(R f, p) .
$$

For $p=(0,0)$, we take the discrete Radon transform over $H((0,1), 0)$. Since $H((0,1), 0)=$ $\{(q, 0) \mid q \in \mathbf{Z}\}$, it follows that

$$
f(0,0)=R f(H(0,1), 0)-\sum_{q=-N, q \neq 0}^{N} d_{N}(R f,(q, 0)) .
$$

REMARK 4.7. We suppose that all $a$ of $H(a, a p)$ in $\underset{1 \leq j \leq j_{N}-1}{\cup} E(N, j)$ are of the forms $(1, \pm l)$ or $( \pm l, 1), l \geq 1$, and $l_{0}$ their maximum. Then we can deduce that $1+2+\cdots+l_{0}+l_{0} \geq N$ (see Fig. 3 for the case of $N=9$ ). Therefore, since $E\left(N, j_{N}\right)=$ $\{H((1,0), \pm N), H((0,1), \pm N)\}$, the maximum $\|a\|$ of $H(a, a p)$ in $\underset{1 \leq j \leq j_{N}}{\cup} E(N, j)$ is $O(\sqrt{N})$. Hence, in the formulas (19) and (20) we use $R f(H(a, k))$ with $\|a\|=O(\sqrt{N})$.

Step4. Let $f$ be an arbitrary function in $\mathcal{S}\left(\mathbf{Z}^{2}\right)$. We extend the definition of the operator Shave $_{N}$ to $f$, that is, we apply (19) to $f$. We note that, in the process to define Shave ${ }_{N}$, each hyperplane $H(a, a p)$ is used to vanish the value $f(p)$ at $p$ when $f$ is supported in $[-N, N]^{2}$. 


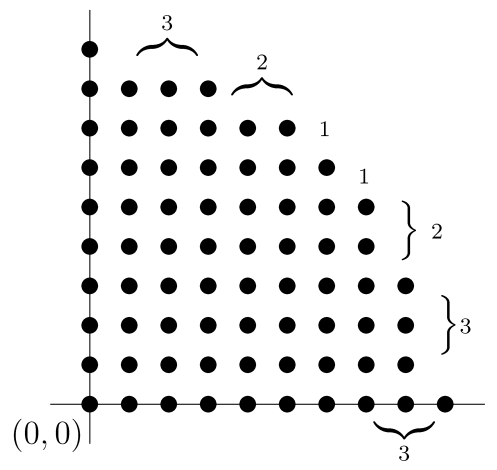

FIGURE 3. $\quad V(9,16)_{+}, j_{9}=17$ and $l_{0}=3$

Therefore, the total number of discrete hyperplanes which appear to define $\mathrm{Shave}_{N}$ is at most $O\left(N^{2}\right)$ and thus, the total number of hyperplanes appeared in (19) is $O\left(N^{3}\right)$. Since $f-f \chi_{N}$ is supported in $B(N)^{c}$ and is rapidly decreasing, it is easy to see that for each $q \in \mathbf{N},|f(m)| \leq$ $C_{q}\left(1+\|m\|^{2}\right)^{-q}$ and thus, $\left\|R\left(f-f \chi_{N}\right)\right\|_{\infty} \leq C_{q} \sum_{m \in B(N)^{c}}\left(1+\|m\|^{2}\right)^{-q} \leq c N^{-2(q-1)}$. Hence, if $q>2$, then

$$
\left\|\operatorname{Shave}_{1} \circ \cdots \circ \operatorname{Shave}_{N}\left(f-f \chi_{N}\right)\right\|_{\infty} \leq C_{q} N^{3} N^{-2(q-1)},
$$

and this goes to 0 if $N \rightarrow \infty$. Hence, it follows that

$$
\begin{aligned}
f(0,0) \delta_{(0,0)} & =\left(f \chi_{N}\right)(0,0) \delta_{(0,0)} \\
& =\text { Shave }_{1} \circ \operatorname{Shave}_{2} \circ \cdots \circ \operatorname{Shave}_{N-1} \circ \operatorname{Shave}_{N}\left(f \chi_{N}\right) \\
& =\lim _{N \rightarrow \infty} \operatorname{Shave}_{1} \circ \operatorname{Shave}_{2} \circ \cdots \circ \operatorname{Shave}_{N-1} \circ \operatorname{Shave}_{N}(f) .
\end{aligned}
$$

Therefore, we can obtain the following.

THEOREM 4.8. Let notations be as above. For $f \in \mathcal{S}\left(\mathbf{Z}^{2}\right)$,

$$
\begin{aligned}
f(p) & =\lim _{N \rightarrow \infty} d_{N}(R(f), p), \quad p \neq(0,0), \\
f(0,0) & =R f(H(0,1), 0)-\lim _{N \rightarrow \infty} \sum_{q=-N, q \neq 0}^{N} d_{N}(R(f),(q, 0)) .
\end{aligned}
$$

ACKNOWLEDGMENT. The author would like to thank the referee for his valuable comments and suggestions to improve the quality of the paper.

\section{References}

[ 1 ] Abouelaz, A. and Ihsane, A. Diophantine Integral Geometry, Mediterr. J. Math. 5 (2008), 77-99. 
[2] Abouelaz, A. and Kawazoe, T. Characterizations of function spaces by the discrete Radon transform, Integral Transforms and Special Functions 23 (2012), 627-637.

[ 3 ] Helgason, S. The Radon transform on Euclidean space, compact two-point homogeneous space and Grassmann manifolds, Acta Math. 113 (1965), 153-180.

[ 4 ] Helgason, S. The Radon transform, Birkhäuser, Boston, 1980.

[ 5 ] RADON, J. Über die Bestimmung von Funktionen durch ihre Integralwerte längs gewisser Mannigfaltigkeiten, Ber. Verh. Sächs. Akad. Wiss. Leipzig, Math.-Nat. Kl. 69 (1917), 262-277.

[6] Strichartz, R. S. Radon inversion-variations on a theme, Amer. Math. Monthly 89 (1982), 377-384, 420-423.

Present Address:

DEPARTMENT OF MATHEMATICS,

KEIO UNIVERSITY AT SFC,

ENDO, FUJISAWA, KANAGAWA 252-0882, JAPAN. 